\title{
Late air embolism with interventional removal after isolated coronary artery bypass grafting
}

U. Engelmann, MD, ${ }^{a}$ H. Minden, MD, ${ }^{b}$ U. A. Stock, MD, ${ }^{a}$ R. Kühnel, MD, and J. M. Albes, MD, ${ }^{a}$ Bernau/Berlin, Germany

$\mathrm{A}$

ir embolization during coronary artery bypass grafting $(\mathrm{CABG})$ is an unpleasant but common phenomenon. Application of extended reperfusion with increased perfusion pressure normally resolves this problem. Late occurrence after successful weaning from extracorporeal circulation and transferal to the intensive care unit has so far not been published. We report on a case of successful interventional removal, highlighting an optimal collaborative approach for the treatment of coronary artery disease.

From the Departments of Cardiac Surgery ${ }^{\mathrm{a}}$ and Cardiology, ${ }^{\mathrm{b}}$ Heart Center Brandenburg, Bernau/Berlin, Germany.

Received for publication Dec 29, 2005; accepted for publication Jan 24, 2006.

Address for reprints: Ulrich Stock, MD, Department of Cardiac Surgery, Heart Center Brandenburg, Ladeburger Strasse 17, 16321 Bernau, Germany (E-mail: Ulrich.stock@charite.de).

J Thorac Cardiovasc Surg 2006;131:1403-4

$0022-5223 / \$ 32.00$

Copyright $\odot 2006$ by The American Association for Thoracic Surgery doi:10.1016/j.jtcvs.2006.01.046

\section{Clinical Summary}

A 67-year-old female patient with triple coronary artery disease was admitted to our institution with acute occlusion of the left anterior descending coronary artery (LAD), unstable angina, and significantly reduced left ventricular function with an estimated ejection fraction of $30 \%$. Preoperative echocardiography revealed normal valve function and intact atrial and ventricular septa. During induction of anesthesia the patient's hemodynamic condition deteriorated, with the resulting necessity of urgent establishment of extracorporeal circulation. She received triple $\mathrm{CABG}$ with vein grafts. Veins were distally anastomosed to the LAD, first marginal (M1), and proximal right coronary artery (RCA). After removal of the aortic crossclamp and subsequent tangential aortic clamping, the RCA and LAD bypasses were proximally anastomosed to the aorta. Owing to severe aortic calcification, the M1 bypass was attached in an end-to-side fashion to the LAD graft. All grafts were thoroughly deaired by punction with a hypodermic needle (28 gauge). The patient was successfully weaned from extracorporeal circulation with a normal electrocardiogram (ECG) (Figure 1, A) and low-dose catecholamines. She was transferred to the intensive care unit without any complications. Two hours after admittance,

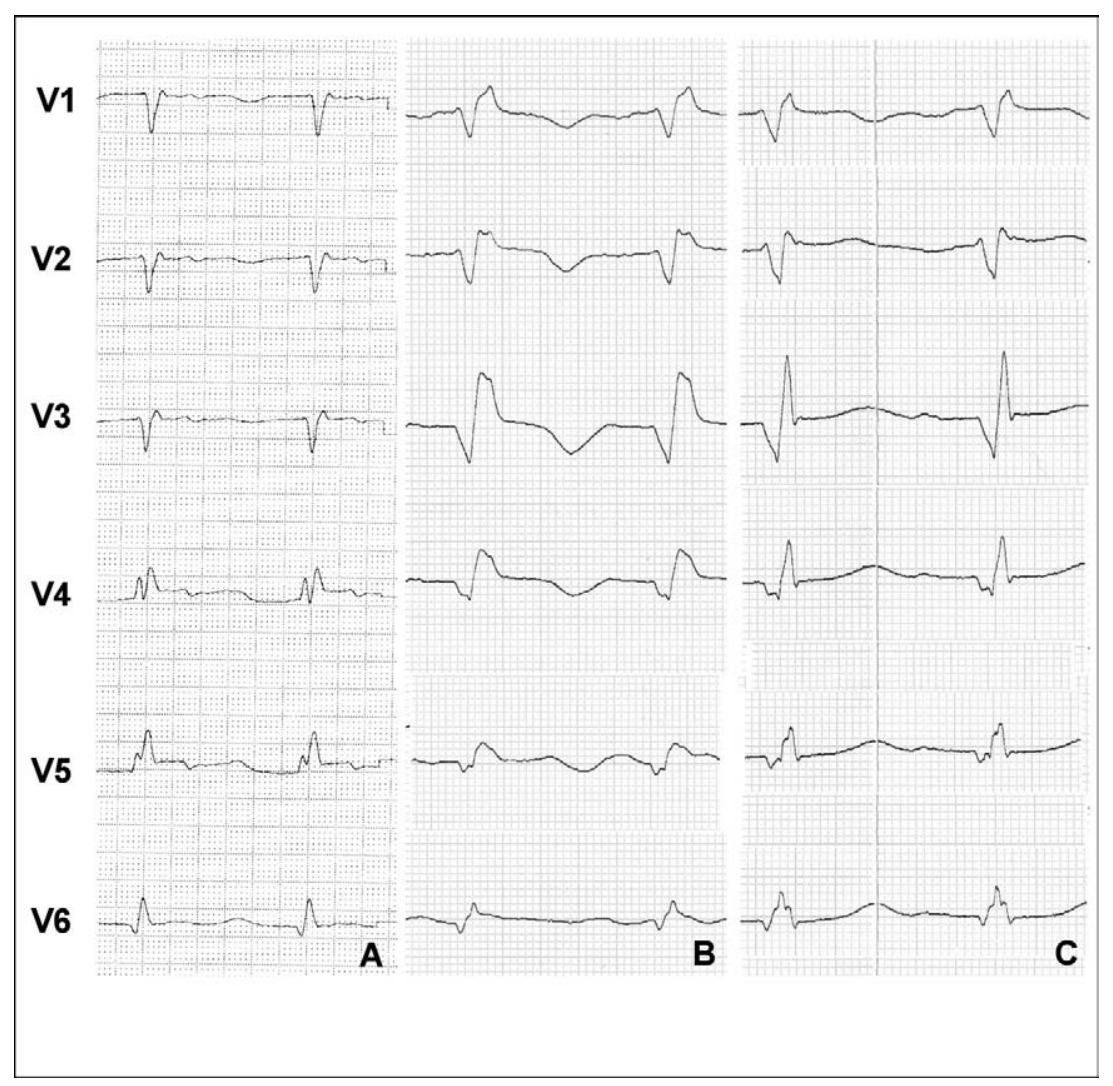

Figure 1. ECG after weaning from bypass (A), before bubble removal (B), and after bubble removal (C). 


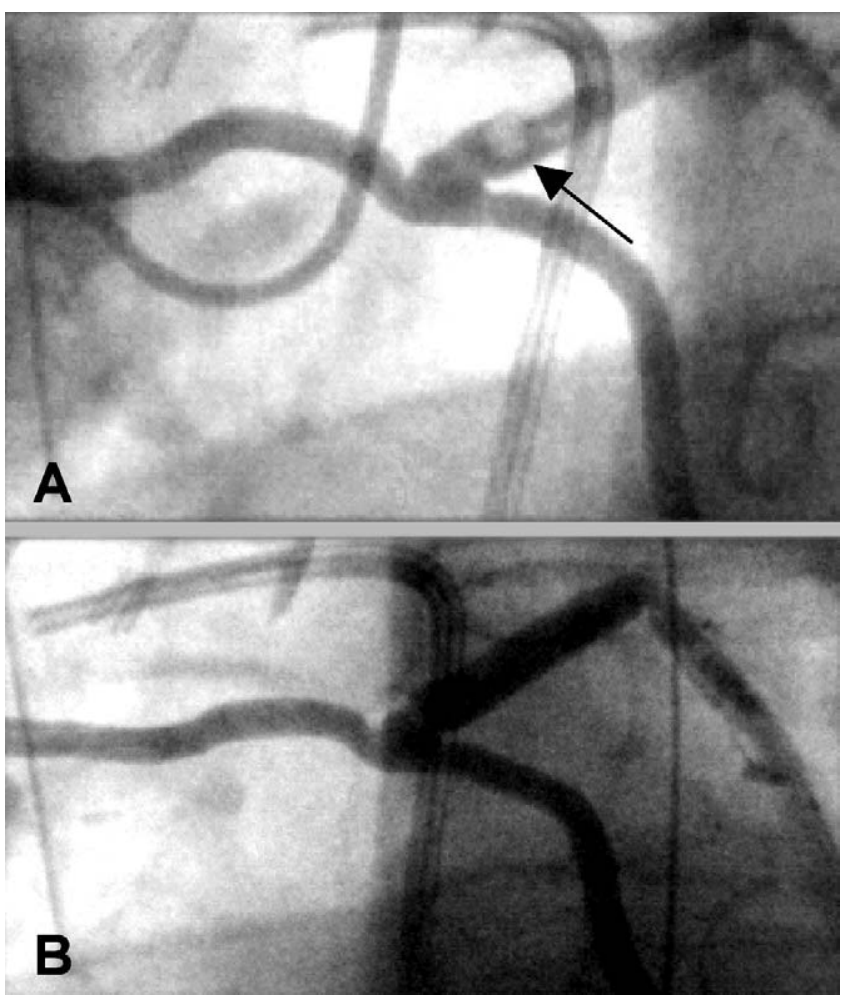

Figure 2. A. Angiogram with air bubble entrapped in the Yanastomosis B. Angiogram after bubble removal.

severe changes developed on the ECG, with ST elevations in $\mathrm{V}_{2}$ through $\mathrm{V}_{4}$, II, III, and aVF (Figure $1, B$ ). With increasing hemodynamic instability and a suspected bypass occlusion, the patient was transferred to the catheter laboratory. Angiography revealed a floating air bubble in the Y-anastomosis of the M1/LAD bypass impairing blood flow of the M1 bypass (Figure 2, A). The bubble was successfully removed with a vacuum extraction catheter (Fig- ure 2, B). ECG changes disappeared immediately with prompt hemodynamic stabilization (Figure 1, C). Postoperative recovery was initially aggravated by severe respiratory insufficiency resulting from chronic obstructive pulmonary disease and temporary psychosis. However, the patient was discharged on the 17 th postoperative day.

\section{Discussion}

Air embolization during interventional catheter intervention ${ }^{1-3}$ and CABG is a common problem. Incomplete deairing of catheters is the main cause in interventional settings. In contrast, several pathophysiologic mechanisms exist, such as inadvertent injection of air for testing of bypass anastomoses, dislocation of remaining air after tangential clamping, and finally trapped air bubbles in ligated vein branches. Prevention strategies include flushing and thorough deairing before completion of the anastomosis. If, however, air embolism has occurred, an increased perfusion pressure and extended reperfusion period normally enable bubble transit through the coronary artery system. Late air embolism after surgery of the coronary arteries mimicking bypass occlusion has so far not been published. The source of the air bubble in the reported case remains unclear. However, trapped air in the region of the $\mathrm{Y}$-anastomosis of bypasses may have contributed to the late air embolism observed. We strongly recommend recatheterization at the earliest possible moment after CABG if clinical signs of compromised bypass flow such as ECG alterations, cardiac enzyme kinetics, and hemodynamic instability appear. This allows eradication of compromising entities before potential thrombotic bypass occlusion.

\section{References}

1. Khan M, Schmidt DH, Bajwa T, Shalev Y. Coronary air embolism: incidence, severity, and suggested approaches to treatment. Cathet Cardiovasc Diagn. 1995;6:313-8.

2. Patterson MS, Kiemeneij F. Coronary air embolism treated with aspiration catheter. Heart. 2005;91:36-7.

3. Solodky A, Birnbaum Y, Assali A, et al. Coronary air embolism treated by bubble apiration. Cathet Cardiovasc Diagn. 2000;49:452-4. 